\title{
Comment l'expérience individuelle rejoint l'expérience collective: une étude en milieu scolaire à partir du travail de François Delsarte ${ }^{1}$
}

Franck Waille*

Académie de Lyon

Gilberto Icle**

Universidade Federal do Rio Grande do Sul

Résumé Ce texte cherche à montrer - à partir d'une étude pratique réalisée dans un établissement scolaire au Brésil - comment l'expérience individuelle de la proprioception peut être envisagée comme une expérience collective. Á cet effet, l'article décrit une expérimentation réalisée à partir d'exercices issus de la pédagogie corporelle de François Delsarte (1811-1871) et établit ses rapports avec l'Éducation Somatique. Sont analysées les réponses des participants à des questionnaires cherchant à transcrire leurs perceptions, notamment concernant la respiration et la sensation de poids. Enfin, le texte présente une réflexion sur le sens que peut prendre la notion de collectif (et de commun) par l'expérience de la proprioception dans le travail en milieu scolaire.

MOTS-CLÉs: Milieu scolaire; Corps; François Delsarte; Éducation Somatique; Proprioception. 
How the individual experience becomes a collective experience: a study on K-12 Education from François Delsarte's work

Abstract This text aims to show, from a practical study developed in a K-12 Education school in Brazil, how the individual experience of proprioception can be assumed as a collective experience. The paper describes an experiment based on exercises from the corporeal pedagogy of François Delsarte (1811-1871), establishing relations with Somatic Education. The answers provided by female and male students who filled out the study questionnaires are analyzed, transcribing their perceptions, remarkably those referring to breathing and weight sensation. Lastly, it is presented a reflection on the meanings that could be gathered from the notion of collective (and in common) based on the experience of proprioception in the school pedagogic work.

KEYWORDS: K-12 education; Body; François Delsarte; Somatic Education; Proprioception.

\section{Como a experiência individual se faz experiência coletiva: um estudo na Educação Básica a partir do trabalho de François Delsarte}

Resumo Este texto procura mostrar - a partir de um estudo prático realizado numa escola de Educação Básica no Brasil - como a experiência individual da propriocepção pode ser tomada como uma experiência coletiva. Com efeito, o artigo descreve uma experimentação realizada a partir de exercícios provenientes da pedagogia corporal de François Delsarte (1811-1871), estabelecendo relações com a Educação Somática. Analisa-se as respostas dadas por alunos e alunas participantes da pesquisa, a partir de questionários, transcrevendo suas percepções, notadamente no que se refere à respiração e à sensação de peso. Enfim, apresenta-se uma reflexão sobre os significados que se poderia depreender da noção de coletivo (e de comum) a partir da experiência da propriocepção no trabalho pedagógico na Escola.

PAlAVRAS-ChAVE: Educação básica; Corpo; François Delsarte; Educação Somática; Propriocepção.

Ce texte étudie la question de l'expérience individuelle et de l'expérience collective au sein du travail pédagogique corporel en milieu scolaire. La recherche que nous avons réalisée a été l'occasion de proposer une transmission d'éléments des enseignements de François Delsarte (1811-1871) auprès d'un public d'adolescents non familiarisé avec l'Éducation Somatique. 
Nous avons proposé à deux groupes d'adolescents, au Colégio de Aplicação, de l'Universidade Federal do Rio Grande do Sul, douze séances sur environ quatre mois. Pour un groupe de onze élèves de 15 ans, nous avons donné une introduction de quarante-cinq minutes au cours de théâtre; pour un groupe de dix élèves de 18 ans, nous avons donné un cours d'une heure et demie associant un temps de préparation et un temps de danse improvisée. Durant les séances, il était proposé aux élèves de verbaliser sur ce qu'ils ressentaient ; nous prenions notes des verbalisations significatives (très affirmatives). Et lors de la dernière séance, un questionnaire a été proposé concernant les ressentis durant ces exercices (pour favoriser des réponses le plus en lien possible avec l'expérience, nous faisions ensemble un exercice, puis un temps était donné aux élèves pour noter leurs observations). Nous avons les données de quinze questionnaires (il y a eu six absents sur les vingt-et-un élèves).

Notre but principal a été d'ouvrir un espace d'expérimentation dans le quotidien d'un établissement scolaire afin de réfléchir sur les perceptions corporelles d'évaluer comment elles peuvent ou non se développer lors d'un travail dans ce milieu. Contrairement à des connaissances plus traditionnelles, la perception est d'habitude mise à l'écart dans les établissements scolaires. En effet, le corps y est négligé bien qu'il soit le locus de conflits politiques et sociaux dans les différents champs de savoir. L'école pourrait-elle profiter des pratiques portant sur le travail perceptif ? Ce travail pourrait-il mettre ensemble les perceptions individuelles, proprioceptives, et l'expérience collective?

Dans la tentative de faire avancer ces questions, nous nous sommes proposés de reprendre une partie du travail de Delsarte, pionnier de la pédagogie corporelle. Nous avons fait appel aussi à des idées et des pratiques surgies les dernières années dans l'Éducation Somatique, en les mettant en relation avec le travail du pédagogue français.

Si les recherches et les enseignements de Delsarte peuvent être considérés comme l'une des sources de l'Éducation Somatique (WAILLE, 2016), d'emblée cet artiste proposait à ses élèves une démarche très large qui, d'une certaine façon, dépassait le cadre de l'Éducation Somatique telle quelle a été définie initialement par Thomas Hanna au début des années 1970.

Pour Hanna en effet, «la somatique est le domaine qui étudie le soma: à savoir, le corps tel que perçu de l'intérieur par une perception à la première personne» $(1986, \text { p. } 4)^{2}$ en intégrant des différentes dimensions de l'individu dans le mouvement (HANNA, 1979, 1986). Si l'Éducation Somatique prend nécessairement en compte les relations interpersonnelles et avec l'environnement (HANNA, 1976, p. 32 ; FERNANDES, 2015, p. 22), elle est d'abord caractérisée par l'exploration personnelle (BATSON, 2009, p. 2).

Or, la dimension somatique des enseignements de Delsarte est synthétisée par cette phrase-clé : «Un élève de ma méthode est un observateur silencieux des phénomènes qui l'entourent et se manifestent au-dedans de lui-même » (PORTE, 1992, p. 260). Il y a alors une double direction de l'observation : vers soi (les phénomènes 
qui «se manifestent au-dedans de lui-même ») et vers le collectif, voire l'universel (les phénomènes qui "l'entourent»). Delsarte a élaboré un enseignement précis fait d'exercices et de lois expressives appuyées sur ses observations. Mais loin de proposer des recettes toutes faites, il demandait à ses élèves de revenir au processus d'observation qui fut le sien, persuadé que l'individuel rejoignait le collectif dans une dynamique de découverte ouverte à l'infini (cf. DELSARTE in WAILLE, DAMOUR, 2015, p. 296-298). Passer par l'observation et l'expérience était pour lui l'étape indispensable pour intégrer des connaissances.

Cette perspective, si elle va au-delà de la définition initiale de l'Éducation Somatique, rejoint en revanche des pratiques aujourd'hui rattachées à la somatique, dans lesquelles l'observation peut alternativement être interne ou tournée vers les objets qui nous entourent (CORRÊA GRAVINA, 2015, p. 239). C'est le cas en particulier du Body-Mind Centering ${ }^{\circledR}\left(\mathrm{BMC}^{\circledR}\right)$, où les observations sur soi participent d'une construction collective de la connaissance de tel ou tel élément du corps humain : l'observation individuelle est donc alors la base de l'élaboration d'une connaissance objective et qui peut être expérimentée par d'autres personnes.

Ainsi, nous allons d'abord présenter brièvement le travail de Delsarte en mettant en valeur sa dimension somatique. Nous préciserons ensuite les dynamiques pédagogiques de l'Éducation Somatique. Nous exposerons enfin notre recherche pratique au sein du Colégio de Aplicação de l'Universidade Federal do Rio Grande do Sul, avec en particulier les deux exercices sur lesquels s'appuie notre étude. L'analyse des retours des élèves permettra de faire ressortir deux éléments ayant pris une dimension collective à caractère plus général: le premier concerne la respiration, et le second la sensation du poids.

\section{François Delsarte, ses enseignements et l'Éducation Somatique}

Autant qu'un chanteur fameux en France, Delsarte fut un enseignant exceptionnel. Sa renommée, européenne de son vivant, vint de ses talents de professeur de chant ou de déclamation, et de sa méthode expressive novatrice : il associait de manière complètement neuve le travail de la voix parlée et chantée et le travail du geste, s'appuyant sur une anthropologie et une métaphysique organisées autour de la Trinité chrétienne. La pertinence de cet enseignement fit qu'il se répandit dans l'ensemble de l'ère occidentale dans la deuxième moitié du XIXe siècle : après avoir progressivement touché la plupart des pays d'Europe de l'Ouest (avant de rejoindre la Russie à la fin du siècle), il a d'abord rejoint l'Australie par l'intermédiaire de Camille Delsarte (frère de François formé auprès de lui et qui émigra en Tasmanie en 1851), puis les États-Unis d'Amérique dès 1870 grâce à l'élève le plus proche du maître, Steele Mackaye (qui se vit dans l'obligation de transmettre lui-même ce qu'il avait appris à Paris, la mort ayant empêché Delsarte de traverser l'Atlantique). Il y eut trois décennies de «Delsartemania » nord-américaine. Ainsi progressivement, d'élèves en élèves d'élèves, le delsartisme (la diffusion vivante des enseignements de ce professeur ayant délibérément choisi de transmettre ses connaissances de maître à disciples) est venu irriguer tant la formation des comédiens (puis des acteurs) de l'Amérique du Nord à la Russie, que la naissance de la danse moderne allemande comme américaine, ou encore léclosion de ce qui est 
maintenant appelé les méthodes d'Éducation Somatique.

Comme beaucoup des futurs somaticiens ${ }^{3}$, Delsarte bâtit ses enseignements à partir d'une problématique personnelle : retrouver sa voix chantée, mise à mal par ses études au conservatoire de Paris. Son expérience de rééducation vocale s'appuie sur des processus d'observation des phénomènes expressifs qui se sont mis en place dès son enfance et qui, vers ses 16 ans, se sont centrés sur les liens entre les gestes et la voix. Cette rééducation a été un temps d'approfondissement l'amenant à descendre aux niveaux anatomique et physiologique - il entreprit des études dans ces domaines. Et ses processus d'observation, de rééducation et d'étude du vivant l'ont amené à prendre en compte les dimensions anthropologique, spirituelle et métaphysique qui sont devenues le cadre conceptuel de son travail de transmetteur.

La première publication de textes de Delsarte dans leur langue d'origine (PORTE, 1992) et les premiers travaux de doctorat basés sur une étude approfondie de ses archives (RANDI, 1992 ; WAILLE, [2009] 2011 ; GAUTHIER, 2011) ont permis de plonger au cœur de ses enseignements. Louvrage La méthode somatique expressive de François Delsarte (2016), qui synthétise la recherche de doctorat de l'un des auteurs de cet article et celles qu'il a menées ensuite (en particulier sur les archives des delsartistes américains Mackaye et Henrietta Hovey, et du canadien-français Thomas-Étienne Hamel), met en valeur cette dimension somatique peu prise en compte jusque-là.

L'axe central du travail de Delsarte est de favoriser l'équilibre entre toutes les dimensions de la personne (vitale, intellectuelle et spiritualo-émotive selon son approche), ainsi que l'harmonie entre le corps et l'âme. Or cela est au cœur de la somatique, le choix de la racine grecque sôma (corps) visant à échapper au dualisme occidental entre le corps et l'âme (ou l'esprit) (HANNA, 1985). Delsarte renvoyait à la notion aristotélicienne selon laquelle « l'âme, considérée dans son essence, est la forme du corps » (AQUIN, 1999, I, p. 665), c'est-à-dire son inséparable principe d'existence. Il traduisait cela par cette formule synthétique : «À chaque fonction spirituelle répond une fonction du corps ; à chaque grande fonction du corps répond un acte spirituel »(DELSARTE, 2006a, n. p.). Cette formule, qui dit les interrelations constantes et réciproques du corps et de l'âme (il convient de lire « spirituel » dans le sens de ce qui renvoie à l'âme, à la psyché), peut être considérée comme l'un des fondements des approches psychosomatiques qui se sont développées depuis.

La dynamique présente chez Delsarte invite à une vision large de la notion de somatique : non seulement liée à ce qui se passe en soi dans le mouvement, mais également dans les phénomènes vocaux et dans la parole articulée ; centrée sur ce qui se passe en soi, mais en concevant cela comme résonnant avec ce qui se passe chez l'autre comme dans l'ensemble de l'Univers ; une approche somatique non seulement vue comme source de connaissance et d'équilibre de soi, mais également comme fondement possible de l'expression artistique, et comme renvoyant fondamentalement à 
une intériorité et à une dimension spirituelle. C'est donc à une démarche somatique contemplative et expressive qu'invitait Delsarte.

\title{
La transmission en Éducation Somatique : objectifs et enjeux pédagogiques
}

L'Éducation Somatique, dans sa dimension centrale de conscience de ce qui se passe en soi dans le mouvement, est d'abord une invitation à une conscience proprioceptive fine. La proprioception, ou sensibilité profonde liée aux informations sensorielles en provenance des capteurs musculaires, tendineux, articulaires et vestibulaires, désigne la perception, consciente ou non, de la position des différentes parties du corps dans la statique comme dans la dynamique ; elle peut être désignée comme « le sens de soi » (LIMA CAETANO, 2015, p. 217) ${ }^{4}$ et envisagée comme « un sens premier indispensable à l'émergence de la conscience de soi en tant qu'être capable d'action" (ROLL, 2003, p. 64). Cette conscience de soi associée au mouvement peut s'affiner et inclure la prise en compte de la respiration (GODARD, 1994 ; ALEXANDER, 1996), ou encore la perception des différents tissus du corps, voire aller jusqu'à la conscience cellulaire (BAINBRIDGE-COHEN, 1993). Au total, l'Éducation Somatique vise à «l'intégration entre la sensation, le sentiment et l'action (COHEN, 1993)» (FERNANDES, 2015, p. 15) .

Les pratiques somatiques sont soit des techniques (basées sur des exercices très organisés), soit des méthodes (basées sur des procédés offrant un cadre expérimental), basées sur des principes définissant de manière générale l'approche somatique (FERNANDES, 2015). Celle-ci peut être vue comme « un contexte d'apprentissage réceptif et perceptif facilitant la connexion sensorielle»(FERNANDES, 2015, p. 13) Plus que sur les contenus eux-mêmes, c'est dès lors sur la manière de transmettre ou de proposer des expérimentations qu'est mis l'accent. Ainsi,

\begin{abstract}
une technique somatique peut être enseignée de manière totalement incohérente, c'est-à-dire non somatique. Ce serait ainsi le cas, par exemple, si on enseignait les Bartenieff Fundamentals ${ }^{\mathrm{TM}} \mathrm{com}^{-}$ me une série d'exercices à réaliser selon une séquence pré-établie et apprise par imitation. Dès lors, la cohérence entre la manière de transmettre et le contenu est une caractéristique de la somatique, qui confirme son caractère intégré (FERNANDES, 2015, p. 17)
\end{abstract}

L'attention à la manière de transmettre autant qu'aux contenus est donc un axe fondamental de toute pratique somatique. La question du temps est alors particulièrement importante : le temps d'entrer progressivement dans la démarche somatique en se familiarisant avec elle au long des séances (CARRINGTON, 2009, p. 57 ; CORRÊA GRAVINA, 2015), mais aussi et d'abord le temps de la séance elle-même, son rythme, son tempo. Le ralentissement du rythme est ainsi un élément caractérisant les pratiques somatiques (BOLSANELLO, 2011). Il vise à aider à « se connecter avec ses rythmes personnels qui, bien souvent, sont perdus ou perturbés dans la vie quotidienne " : il s'agit alors d'une " redécouverte de ses propres rythmes » (FERNANDES, 2015, p. 22) ${ }^{8}$. La question du rythme est dès lors très subjective, dans la mesure où elle est à ajuster avec la pratique des participants, avec leur familiarité avec 
la proprioception et avec leur capacité à explorer à partir de leurs sensations, sachant que chaque personne a son propre rythme. Avec un public d'adolescents, entrer dans la dimension somatique est un objectif pédagogique en lui-même, et la question du ralentissement du rythme qui lui est associé peut être un défi : il s'agit tout à la fois de favoriser une écoute proprioceptive en ralentissant le rythme, mais sans lasser le public auquel s'adresse la pratique (un rythme trop lent peut vite devenir ennuyeux et vide pour qui n'y voit pas de sens).

La question du rythme renvoie à l'axe de réflexion de cet article : est-il possible de passer de l'expérimentation individuelle fondée sur ses propres sensations, à une expérience collective ? Il y a dans la méthode de Delsarte une tension dynamique entre des exercices et des lois expressives proposés par l'enseignant, et la démarche demandée aux élèves de tout re-expérimenter et de tout ré-observer afin de vérifier et d'intégrer ces connaissances. Comme pour toute pratique somatique, enseigner des éléments de cette méthode implique une certaine manière de transmettre afin de favoriser la perception intérieure. Il est possible, par exemple, de proposer des exercices de manière relativement répétitive afin de permettre de revenir patiemment sur ses sensations (ou sur ses absences de sensations), sans que cela soit fait mécaniquement, afin de garder une dynamique vivante et pouvant alimenter le travail créatif. Pour le transmetteur, il convient d'être à l'écoute de ce qui se passe en soi pour aider les autres à l'être aussi, et d'installer un climat de type méditatif (au rythme ralenti) favorable au développement de la perception intérieure. Il s'agit également, par un questionnement verbal ouvert, de faire appel à tous les sens pour tenter d'amener à une prise de conscience qu'il se passe quelque chose en soi, sans donner de réponse sur ce qui se passe. Les participants sont alors amenés à porter leur attention sur ce qui se passe en eux, au niveau physique d'abord, puis à un niveau plus intérieur/psychologique (dans quel état cela m’amène ?...) et, éventuellement, à un niveau plus général de ressenti : quelle "ambiance ", " atmosphère » se dégage quand on travaille tel élément de soi de telle manière?

\section{La recherche pratique}

Entre mars et juillet 2017, en collaboration avec la professeure de théâtre Carmen Soares du Colégio de Aplicação da Universidade Federal do Rio Grande do Sul, nous avons donné douze séances à chacun de ces groupes : a) un groupe de première année d'enseignement secondaire (onze élèves de 15 ans), dans le cadre des cours obligatoires de théâtre : nous assurions la première partie du cours (quarantecinq minutes) par un travail de préparation corporelle, qui ensuite était réinvesti dans des exercices d'improvisation théâtrale centrés sur telle ou telle partie du corps, conçus et menés par la professeure Carmen Soares; b) un groupe de dernière année d'enseignement secondaire (dix élèves de 18 ans), dans le cadre d'un cours optionnel orienté vers la danse improvisée intitulé " Expresse-se - Movimente-se - Liberte-se " : nous assurions l'ensemble du cours (une heure et demie) par un travail de préparation corporelle qui s'enchainait avec des propositions d'improvisation dansée.

Dans les deux cas, il s'agissait d'adapter des éléments de la méthode de Delsarte avec, au-delà d'objectifs spécifiques à chaque groupe, un objectif commun à dimension somatique : favoriser la prise de conscience de ce qui se passe en soi lors 
des mouvements, et permettre à partir de cela une utilisation consciente et expressive du corps. Autrement dit, il s'agissait de développer la conscience du « corps perçu de l'intérieur »(HANNA, 1990-91) ${ }^{10}$ dans une visée expressive.

\section{Exercices à la base de notre analyse somatique}

Pour tenter d'évaluer le développement de la prise de conscience de la dimension somatique et voir si l'expérience individuelle pouvait rejoindre le collectif, nous avons choisi de nous centrer sur les premiers exercices proposés aux deux groupes: a) l'intégration consciente de la position "genoux libres ", avec les spécificités que cette position amène au niveau de la respiration, de l'organisation du rachis (WAILLE, 2016, p. 252-257) et de l'activité musculaire des jambes (donnée amenée par un élève, nous y reviendrons) ; b) le travail de décomposition des parties du corps, c'est-à-dire de relâchement de chaque section corporelle à partir des articulations en la laissant pendre de tout son poids, avec les sensations que cela amène dans les parties travaillées (WAILLE, 2016, p. 264-304).

\section{Les trois positions des genoux et leurs répercussions dans l'organisme}

Nous proposions d'intégrer la position libre des genoux en la comparant

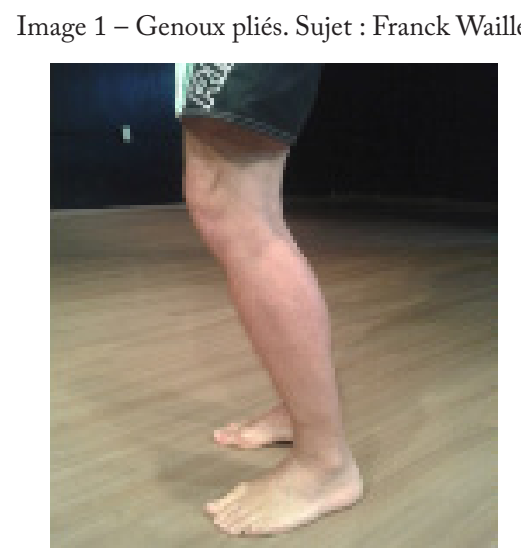

Photographie : Carmen Soares. Porto Alegre, 2017. 
Comment l'expérience individuelle rejoint l'expérience collective: une étude en milieu scolaire à partir du travail de François Delsarte

Image 2 - Genoux fermés, bloqués. Sujet: Franck Waille

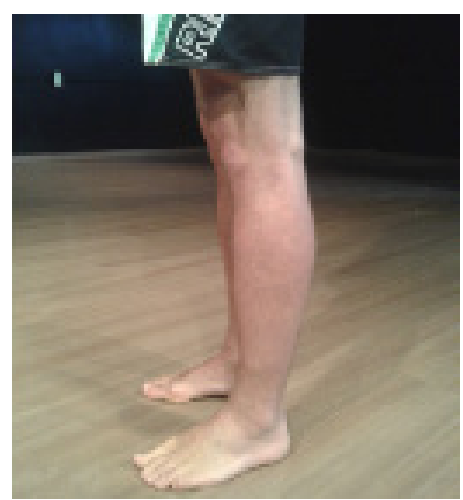

Photographie : Carmen Soares. Porto Alegre, 2017.

Image 3 - Genoux libres. Sujet : Franck Waille

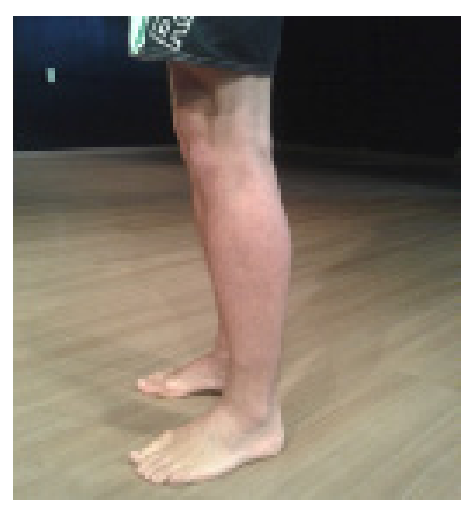

Photographie : Carmen Soares. Porto Alegre, 2017.

Tableau 1 - Grille comparative des sensations généralement associées aux positions des genoux

\begin{tabular}{|l|l|l|l|}
\hline \multirow{3}{*}{ Genoux pliés } & $\begin{array}{l}\text { Organisation du } \\
\text { rachis }\end{array}$ & Respiration & $\begin{array}{l}\text { Activité musculaire } \\
\text { des jambes }\end{array}$ \\
\cline { 2 - 4 } & $\begin{array}{l}\text { Bon alignement, } \\
\text { fluidité }\end{array}$ & $\begin{array}{l}\text { Complète, allant } \\
\text { jusqu'à la respiration } \\
\text { basse }\end{array}$ & Importante \\
\hline
\end{tabular}

\begin{tabular}{|l|l|l|l|}
\hline \multirow{2}{*}{$\begin{array}{l}\text { Genoux } \\
\text { bloqués }\end{array}$} & $\begin{array}{l}\text { Organisation du } \\
\text { rachis }\end{array}$ & Respiration & $\begin{array}{l}\text { Activité musculaire } \\
\text { des jambes }\end{array}$ \\
\cline { 2 - 4 } & $\begin{array}{l}\text { Blocages lombaire } \\
\text { (tendance à } \\
\text { l'hyperlordose) et cervical }\end{array}$ & $\begin{array}{l}\text { S'arrête au niveau du } \\
\text { plexus solaire }\end{array}$ & Faible \\
\hline
\end{tabular}

\begin{tabular}{|l|l|l|l|}
\hline \multirow{2}{*}{ Genoux pliés } & $\begin{array}{l}\text { Organisation du } \\
\text { rachis }\end{array}$ & Respiration & $\begin{array}{l}\text { Activité musculaire } \\
\text { des jambes }\end{array}$ \\
\cline { 2 - 4 } & $\begin{array}{l}\text { Bon alignement, } \\
\text { fluidité }\end{array}$ & $\begin{array}{l}\text { Complète, allant } \\
\text { jusqu'à la respiration } \\
\text { basse }\end{array}$ & Faible \\
\hline
\end{tabular}


à deux autres: genoux fléchis et genoux fermés (ou bloqués). Sous les photographies illustrant ces trois positions, nous indiquons les sensations qui leurs sont généralement associées chez les divers participants de nos cours et stages (dans les commentaires que nous ferons plus bas, nous désignons cela par « cadre de référence »):

La position " genoux libres » est la synthèse vertueuse des deux autres : avec la taille complète de la personne, elle permet une faible activité musculaire (les structures osseuses alignées jouent tout leur rôle dans l'organisation de la verticalité), une organisation du rachis fluide et une respiration ample. Elle est une excellente prédisposition au mouvement, dans une qualité de fluidité ou, pour reprendre les mots de Delsarte définissant la qualité du mouvement qu'il désirait transmettre, d'« union de la force à la suavité » (2006b, n. p.).

Notons que si elle semble proche de la posture qu'Eugenio Barba décrit comme étant la posture idéale des genoux acquise par les acteurs de l'Odin Teatret, permettant l'impulsion d'une action qui s'ignore encore et qui pourrait prendre n'importe quelle direction, elle est en fait différente : dans la proposition de Barba, les genoux sont légèrement pliés, comme dans le tennis ou l'escrime par exemple, quand on doit être prêt à réagir (1994, p. 19-20). Dans la position libre des genoux de Delsarte, il n'y a pas de pliement mais seulement un léger avancement de l'articulation à partir de la position genoux bloqués (ou une extension à partir de la position genoux pliés).

Le constat fait généralement est que quand nous sommes à l'arrêt, nous adoptons plutôt la position des genoux bloqués vers l'arrière, position qui est de loin la moins intéressante. Si nous suivons les analyses de Delsarte, cette position est le fruit des habitudes acquises, alors que la position libre des genoux est plutôt celle des petits enfants - il recommandait d'observer les manières de bouger des enfants, car ils sont plus proches de fonctionnements non influencés par les habitudes individuelles ou culturelles (WAILLE, 2016, p. 264). Tout le travail consiste à lentement intégrer la position libre des genoux à l'arrêt. Après avoir pris conscience de cette position, une manière de l'intégrer est de marcher, de s'arrêter, de constater dans quelle position sont placés nos genoux et, si nécessaire, d'adopter la position libre. Une autre manière est, dès que l'on est amené à être debout, par exemple pour un travail corporel (comme dans les exercices de décomposition décrits ci-après), de prendre un court moment pour vérifier la position de ses genoux.

\section{La décomposition/recomposition des différentes parties du corps}

Strictement, la décomposition est le fait de pouvoir, dans un mouvement, laisser pendre de tout son poids chaque partie du corps inutilisée afin d'assurer le maximum de souplesse au geste, selon cette loi du geste : « Les articulations se déploient successivement et harmonieusement, toute articulation qui n'a pas agi doit demeurer absolument pendiculaire sous peine de raideur » (DELSARTE, dictée de 1869, n.p.).

Les exercices préparatoires individualisent chaque articulation, et la position debout permet de laisser travailler au maximum le poids dans chacune de ces articulations (il ne s'agit pas de tirer sur les tissus pour les allonger, comme cela est fait 
par exemple dans le streatching, mais de laisser uniquement jouer le poids qui, progressivement, libère les tissus de leurs tensions). Ils s'apparentent à la relaxation, mais

Image 4 - Décomposition et recomposition de la main. Sujet: Franck Waille

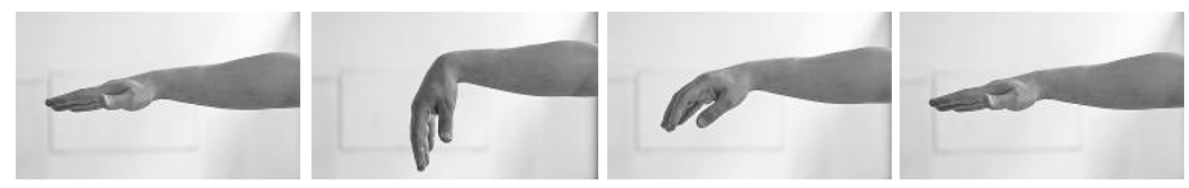

Photographies CDavid Venier. Lyon, 2012

avec une attention plus particulièrement portée sur l'articulation et non sur le muscle (comme c'est généralement le cas dans la relaxation), ce qui permet de relâcher non seulement le muscle mais aussi les tendons et les ligaments. La recomposition est le chemin inverse : une articulation relâchée va retrouver de l'énergie et être mobilisée. L'exercice suivant de la main utilise ces deux moments :

La respiration accompagne ce travail. L'expiration est associée au moment de la décomposition car elle facilite le relâchement de la musculature superficielle et aide la personne à abandonner son poids à la gravité. L'inspiration précède et soutient l'arrivée du tonus dans la partie du corps mobilisée par la recomposition. Cela est une préparation à la dynamique expressive que nous trouvons chez Delsarte : inspiration geste - parole ou chant (WAILLE, 2016, p. 202).

\section{Collecte des informations et analyse des résultats}

Les verbalisations faites par les participants ont permis de voir si une modification de la conscience somatique se faisait chez eux.

Concernant les exercices des genoux, il était suggéré de porter son attention sur ce qui se passait au niveau de la respiration et de l'organisation du rachis, puis de l'activité musculaire des jambes. Les deux premières séances n'ont été accompagnées d'aucune verbalisation, signe que ce type de questionnement et d'attention était nouveau pour les élèves. Lors de la troisième séance, deux élèves exprimèrent sentir une différence dans la respiration et dans le dos, sans pouvoir préciser clairement ces sensations. Lors de la cinquième séance, Gabriel fut le premier à partager un ressenti clair : quand ses genoux sont pliés ou libres, il ressent sa respiration plus large et son dos plus libre. Lors de la septième séance, Guiseppe exprima ressentir une modification de l'activité musculaire de ses jambes en fonction de la position des genoux (tension avec ses genoux pliés). Cette remarque est intéressante parce qu'initialement, nous n'avions pas prévu de prendre en compte la tension musculaire, et nous n'en avions donc pas parlé (nous l'avons fait ensuite). Par son attention à ce qui se passait en lui lors des mouvements, Guiseppe a donc développé une capacité de prise de conscience proprioceptive autonome de l'enseignant. Or, l'Éducation Somatique vise précisément à " susciter un processus étendu de compréhension pour l'éveil des sens, afin d'aller audelà de la vision et de l'apprentissage par la reproduction et la répétition »(PIZARRO, CUNHA, 2017, p. 22) ${ }^{11}$. Lors de cette septième séance, Guiseppe exprima aussi res- 
sentir une respiration plus légère (« leve ») quand ses genoux étaient bloqués. Il précisa ses perceptions respiratoires lors de la neuvième séance, lors de laquelle il exprima ressentir une respiration plus large quand ses genoux étaient libres.

Dans les exercices de décompositions pour les bras et les jambes, l'invitation était de ressentir l'état corporel du membre « décomposé » en le comparant à celui de l'autre membre. Il y eut peu de verbalisations lors des premières séances, et aucune significative. En revanche, dès la première séance concernant le travail des jambes (neuvième séance du cycle), Brenda exprima que la jambe non décomposée « fait mal », et d'autres élèves ressentirent le membre décomposé comme étant plus chaud.

$\mathrm{Au}$ total, les verbalisations indiquent l'émergence et le lent développement de la conscience somatique des élèves. Cela a été précisé par les réponses au questionnaire final.

\section{La respiration et le rapport au poids, domaines d'expérience collective selon les réponses aux questionnaires}

Les réponses aux questionnaires font ressortir deux dimensions en lien avec la problématique du passage de l'expérience individuelle à une expérience collective :

Tableau 2 - Les réponses aux questionnaires concernant les trois positions des genoux

\begin{tabular}{|c|c|c|c|}
\hline $\begin{array}{l}\text { Quand mes genoux } \\
\text { sont... }\end{array}$ & Ma respiration est... & Mon dos est... & $\begin{array}{l}\text { Les muscles de mes } \\
\text { jambes sont... }\end{array}$ \\
\hline bloqués & $\begin{array}{l}\text { Normale (10) } \\
\text { Reposée } \\
\text { Haletante } \\
\text { Facile } \\
\text { Difficile } \\
\text { Lourde }\end{array}$ & $\begin{array}{l}\text { Droit (10) } \\
\text { Normal (3) } \\
\text { Forcé } \\
\text { Inconfortable }\end{array}$ & $\begin{array}{l}\text { Forcés (4) } \\
\text { Contractés (3) } \\
\text { Légers (3) } \\
\text { Normaux (3) } \\
\text { Tendus } \\
\text { Rigides } \\
\text { Lourds } \\
\text { Allongés }\end{array}$ \\
\hline libres & $\begin{array}{l}\text { Normale (9) } \\
\text { Meilleure } \\
\text { Relaxée } \\
\text { Plus ou moins } \\
\text { lourde } \\
\text { Courte } \\
\text { Haletante }\end{array}$ & $\begin{array}{l}\text { Détendu (5) } \\
\text { Normal (4) } \\
\text { Droit (3) } \\
\text { Libre } \\
\text { Plus ou moins } \\
\text { courbé } \\
\text { On dirait que mon } \\
\text { dos est en train de } \\
\text { devenir droit }\end{array}$ & $\begin{array}{l}\text { Détendus (5) } \\
\text { Bons } \\
\text { Confortable } \\
\text { Un peu forcées } \\
\text { Libres } \\
\text { Entre deux }\end{array}$ \\
\hline
\end{tabular}


Comment l'expérience individuelle rejoint l'expérience collective: une étude en milieu scolaire à partir du travail de François Delsarte

Suite du tableau 2

\begin{tabular}{|l|l|l|l|}
\hline pliés & Normale (4) & Détendu (2) & Forcés (8) \\
& Haletante (3) & Courbé (2) & Pesants (3) \\
& Plus lourde (3) & Tordu & Douloureux (3) \\
& Légère & Doutiennent le poids \\
Inconfortable & Posé & du corps \\
& Calme & non agressé & \\
& Difficile d'inspirer & $\begin{array}{l}\text { Douloureux } \\
\text { agressé }\end{array}$ & \\
& Forcé \\
& Fait mal \\
& Moyennement lourd & \\
& & Plus lourd & \\
& & & \\
& &
\end{tabular}

la respiration pour les exercices des genoux ; la question du poids et de la légèreté pour la décomposition des jambes et des bras. Dans les eux cas, cette expérience collective a un caractère général (universel).

\section{Peu de perception des modifications de la respiration en fonction des positions des genoux}

Il y a généralement plus de quinze réponses par case dans la mesure où les élèves ont, la plupart du temps, inscrits plusieurs adjectifs pour faire une même réponse. Parfois, il y a moins de réponses (cas de l'activité musculaire avec les genoux libres).

Les indications « normal » rendent généralement compte d'absence de sensation particulière : elles sont majoritaires concernant la respiration associée aux positions bloqués et libres des genoux ; elles sont présentes mais minoritaires concernant l'organisation du dos associée aux différentes positions des genoux. Elles indiquent alors une moindre conscience proprioceptive. Concernant l'activité musculaire dans la position genoux bloqués, elles sont en revanche cohérentes avec la logique de cette position (ce sont les os qui assurent alors l'essentiel de la position verticale, et non les muscles qui ont une activité minimale).

Les réponses concernant l'organisation du dos et l'activité musculaire des jambes associées aux différentes positions des genoux montrent des perceptions différentielles assez marquées.

Pour le dos, nous avons des réponses qui rejoignent très majoritairement le cadre de référence : dans la position genoux fermés, le dos est ressenti comme étant droit (comprendre raide), forcé ou inconfortable par $80 \%$ des élèves ; dans la position genoux pliés, les notions de détente (détendu, non agressé, posé) et de courbures (dos courbé, rond, tordu) font $53 \%$ des réponses, et elles font $46 \%$ des réponses pour les genoux libres. Mais nous trouvons aussi dans chacun des cas des indications opposées (le dos est ressenti comme inconfortable douloureux ou droit dans $27 \%$ des réponses pour les genoux pliés comme pour les genoux libres). 
Pour l'activité musculaire des jambes associée aux différentes positions des genoux, les renvois à une grande tension musculaire sont très majoritaires pour les genoux bloqués (muscles sentis comme forcés, contractés, tendus ou rigides dans $53 \%$ des réponses), ce qui est différent du cadre de référence. Une grande tension musculaire est majoritairement ressentie pour les genoux pliés (muscles sentis comme forcés, douloureux, très actifs car soutenant le reste du corps dans $80 \%$ des cas), ce qui est revanche en cohérence avec le cadre de référence.

Notons que certains termes sont d'interprétation difficile : muscles légers, entre deux ou lourds, dos lourd.

Concernant la perception des modifications de la respiration en fonction des différentes positions des genoux, les réponses indiquent que cette perception est minoritairement présente quand les genoux sont bloqués ou libres; les réponses sont plus significatives de perceptions différentielles pour les genoux pliés, où la notion de respiration plus lourde peut être comprise comme une respiration profonde, incluant la respiration abdominale. Mais il apparaît bien qu'il peut y avoir des différences complètes de perception d'une personne à l'autre, parfois totalement opposées (par exemple pour les genoux bloqués, nous trouvons une respiration reposée chez l'un et haletante chez l'autre, facile ici et difficile là).

Ce qui ressort globalement concernant la respiration, c'est la difficulté à avoir une perception de ses modifications selon les modifications des positions des genoux (d'où les indications «normale » très majoritairement présentes) : sur ce point, l'expérience collective se fait de manière négative. Elle existe bien néanmoins, et nous allons essayer de la comprendre.

Si l'on se réfère à la différence entre usage (volontaire) et fonctionnement (involontaire) du corps que Mathias Alexander mettait en relation (CORRÊA GRAVINA, 2015, p. 241), il apparaît clairement que parmi les trois paramètres sur lesquels il était demandé aux élèves de porter leur attention (respiration, organisation du dos, activité musculaire des jambes), la respiration est celui qui relève le plus du fonctionnement involontaire puisque c'est un processus automatique vital, assuré par le système nerveux autonome. Développer une conscience fine se rapportant à ce processus demande du temps, l'Éducation Somatique impliquant de « sortir de ses habitudes perceptives, motrices et conceptuelles » (DANIS BOIS cité in BOLSANELLO, 2010, p. 12 $)^{12}$ : or plus un processus est automatique, plus il est difficile d'en développer une perception nouvelle. Chez les adolescents avec lesquels nous avons travaillé, qui découvraient sans doute pour la plupart ce type de direction de l'attention, ce processus semble donc tout juste avoir été amorcé. Dans une perspective d'Éducation Somatique, la conscience des processus respiratoires est essentielle dans la mesure où l'usage de la respiration comme support du mouvement est un élément caractérisant les méthodes somatiques (BOLSANELLO, 2011). La respiration peut alors jouer plusieurs rôles : moyen pour aider à abandonner son poids à la gravité avec une conscience de l'expiration ; moyen d'avoir un feedback proprioceptif sur son état du moment (plus ou moins clame ou stressé), un peu comme un indicateur ; moyen d'aborder sa tridimensionnalité corporelle de l'intérieur en éveillant sa conscience à l'alternance entre « 
Comment l'expérience individuelle rejoint l'expérience collective: une étude en milieu scolaire à partir du travail de François Delsarte

se remplir » et « se vider » etc. (CAMPIGNION, DENYS-STRUYF, 2012 ; ORTIZ, ROUQUET, CAMPIGNION, CORNUT, 2012). C'est donc un phénomène riche et complexe.

La dimension de processus involontaire et la complexité des données mises

Tableau 3 - Les réponses aux questionnaires concernant la décomposition des jambes et des bras

\begin{tabular}{|l|l|}
\hline Sensations de la jambe travaillée & Sensations de la jambe non travaillée \\
\hline Légère (9) & Lourde (6) \\
Relaxée (6) & Dure (6) \\
Confortable (2) & Avec douleur (5) \\
Douce (2) & Forcée (3) \\
Moins fatiguée & Fatiguée (3) \\
Disposée & Rigide \\
Libre & Contractée \\
Un peu endormie & \\
Plus petite & \\
\hline
\end{tabular}

\begin{tabular}{|l|l|}
\hline Sensations du bras travaillé & Sensations du bras non travaillé \\
\hline Léger (9) & Pesant (9) \\
Relaxé (4) & Fatigué (2) \\
Libre (5) & Normal (2) \\
Moins forcé (2) & Un peu forcé \\
Étiré (2) & Moins confortable \\
Reposé & Rigide \\
Plus grand & Dur \\
Abandonné & Moins relaxé \\
C'est possible de tout sentir & Je le sens plus que l'autre \\
\hline
\end{tabular}

en jeu par la respiration expliquent sans doute cette expérience individuelle devenue collective : douze séances (non centrées sur cette seule dimension) ne permettent que le début d'une prise de conscience des modifications fines des réalités respiratoires en fonction des modifications de la position debout induite par les différentes positions des genoux.

\section{Sensation paradoxale de légèreté associée au poids dans la décomposi- tion des jambes et des bras}

Les comparaisons entre les sensations de la jambe décomposée et celles de l'autre montrent des perceptions différentielles très claires. Il en est de même pour les bras.

Et il y a une cohérence presque complète de l'ensemble des réponses : les membres travaillés sont perçus comme plus légers, libres, décontractés, doux, plus grands etc. dans presque la totalité des réponses (seule exception : la mention d'une jambe perçue comme plus petite). Notons la belle variété des adjectifs et expressions 
utilisés pour couvrir le même champ lexical. Les membres non travaillés sont majoritairement ressentis comme pesants, raides, forcés voire douloureux (ce qui peut se comprendre pour la jambe d'appui qui, alors que l'on travaille l'autre jambe, supporte pendant un moment l'ensemble du poids du corps).

Ces réponses contiennent une dimension inattendue : l'association de la notion de légèreté avec les membres décomposés, et celle de lourdeur avec les membres non travaillés. Associations inattendues car un membre décomposé pendant de tout son poids, il est donc physiquement plus pesant. Cela est l'inverse pour un membre non décomposé. La perception ici nest donc pas liée à la réalité physique : ce qui est pesant est ressenti comme léger, et ce qui est tendu est ressenti comme lourd. Deux phrases de Delsarte peuvent éclairer ce paradoxe : « Poids. Ce qui est pesant et léger provient de ce qui n’a ni pesanteur ni légèreté. Certains Pythagoriciens appelaient le vide la matière des corps » (2006c, n.p.) ; et : « Si dans l'expression d'un principe on n'a pas compris son antipode, on n'a rien compris - chaque expression élémentaire porte en elle son antipode » (2005, n.p.).

Les élèves expérimentent donc ces principes qui peuvent sembler abstraits, mais qui se révèlent être ici très concrets et très accessibles par l'expérience. Ils touchent, avec cette association poids/légèreté, à l'un des fondamentaux du travail du mouvement dansé (Godard, 1990 \& 1992), à savoir la dynamique verticale qui sollicite une double action : donner son poids au sol (yield en $\mathrm{BMC}^{\circledR}$ ) pour ensuite se repousser / donner une direction dans l'espace (autograndissement ou projection spatiale en Laban Movement Analysis) (Harbonnier, Dussault, Ferri, article soumis). Autrement dit, il s'agit de donner du poids pour avoir de la légèreté, ce qui peut se comprendre comme un phénomène yin/yang.

Le travail de décomposition, mais aussi la position libre des genoux ${ }^{13}$, favorisent la perception du poids. Nous pouvons utiliser l'expression « sensation du poids / sensation de soi » en écho aux associations que Delsarte faisait avec le poids (unité, moi profond, union des opposés etc.) (WAILLE, 2016, p. 250-252). Ceci trouve un écho dans la notion de "sens de soi " (LIMA CAETANO, 2015, p. 217) ${ }^{14}$ associée à la proprioception.

La prise de conscience du poids en soi, de son action potentielle et des sensations qu'il peut procurer, est le début de la possibilité de pouvoir utiliser ce poids dans le mouvement. Cela est un objectif de la méthode de Delsarte, comme de l'Éducation Somatique en général. Pour Delsarte en effet, « c'est le poids qui fait le mouvement "(MACKAYE, 2006, n.p., p. 23). Et l'une des caractéristiques des méthodes d'Éducation Somatique est la recherche du juste effort dans le mouvement (BOLSANELLO, 2011). Or, plus on utilise le poids dans le mouvement, moins il est nécessaire de produire un effort musculaire grâce au jeu poids/légèreté décrit plus haut. Par ailleurs, Hanna précise que l'approche somatique associe la prise de conscience des phénomènes proprioceptifs et deux éléments fondamentaux dans la conduite du mouvement : la relation gravitaire et l'orientation de l'attention (1987-88). Le focus sur l'utilisation du poids dans le travail de Delsarte est dès lors un élément favorisant la prise de conscience des phénomènes proprioceptifs, donc l'approche somatique. 
Comment l'expérience individuelle rejoint l'expérience collective: une étude en milieu scolaire à partir du travail de François Delsarte

\section{Conclusion}

Notre analyse montre que le progrès de la conscience somatique des élèves est un processus en cours chez eux. Comme le rappelle Walter Carrington, unes des principales références dans l'enseignement de la Technique Alexander, le développement de cette conscience somatique « est définitivement une question de temps; de temps donné par le professeur, de temps que se permet l'élève » $(2009, \text { p. } 57)^{15}$. Les douze séances de travail sont donc une amorce d'un processus qui mériterait de se prolonger. Notre pari est que ce qui a été amorcé se poursuivra chez les participants, même en dehors d'un cadre porteur. Nous pensons en particulier à une attention à la position de ses genoux dans la vie quotidienne.

Les écarts plus ou moins importants avec ce que nous avons appelé le cadre de référence sont des indicateurs de ce processus. Dans une perspective delsartienne, les progrès de la conscience somatique devraient pouvoir combiner la variété des expressions individuelles, et le respect des grandes dynamiques de ce cadre de référence. Celui-ci n'est pourtant pas rigide, mais susceptible d'évoluer, ou plutôt d'être affiné par l'approfondissement du processus d'observation (WAILLE, 2011, chapitre 1). Ainsi l'expérience individuelle peut-elle prendre place dans une expérience collective de manière dynamique et vivante, expérience collective elle-même très ouverte.

Ainsi, les trois positions des genoux expérimentées durant les séances sont-elles à mettre en lien avec les dynamiques excentrique, concentrique et normale (ou équilibrée) qui forment la base du travail somatique expressif delsartien, et qui renvoient à l'archétype trinitaire (Père, Fils, Souffle divin), celui-ci se retrouvant partout selon Delsarte (par exemple dans la trilogie biblique mesure, nombre, poids - Sg, 11, 20). Cela fait écho au sens de soma dans la tradition des Védas hindous (qui va bien au delà du corps comme en grec). Il y désigne d'abord une boisson sacrée (GRIFFITH, 1896, p. 368-420), et « la force motrice pour l'intellect et aussi la réserve de la connaissance scientifique pour les recherches pouvant permettre d'améliorer la prospérité de la société » (FERNANDES, 2015, p. 14) ${ }^{16}$. Dans cette tradition, soma désigne aussi « une divinité et une boisson responsable de l'immortalité, en plus d'une substance constitutive de tous les êtres vivants » (FERNANDES, 2015, p. 14) ${ }^{17}$. Il y a donc, dans les racines du mot somatique comme dans l'approche delsartienne, des liens fondamentaux entre l'expérience individuelle et la dimension collective. Modestement, notre expérimentation a pu être le départ de ce type d'expérimentation et des observations qui l'accompagnent pour les jeunes qui s'y sont prêtés.

En effet, cette expérimentation nous montre la possibilité de penser l'expérience de la proprioception à partir d'une expérience collective. Ainsi, la variété des sensations associées aux différentes positions des genoux, qu'elles soient en lien ou en contradiction avec le cadre de référence, montre le développement d'une conscience de ce qui est vécu lors d'un mouvement. Il est probable qu'un échange verbal entre les participants après les réponses faites à l'écrit dans les questionnaires, aurait apporté des précisions sur tel ou tel terme utilisé pour décrire son expérience, et que des rencontres auraient été possible entre différents termes utilisés par les uns et les autres, dans la mesure où nous ne donnons pas tous le même sens aux mots (la sensation 
de dos «lourd » par exemple, rencontrerait-elle celle de dos détendu, ou celle de dos douloureux ?). En ce sens, résulte de notre réflexion l'idée qu'il est possible d'entendre collectif comme l'expérience du commun, et dans le cas présent, l'idée que des jeunes en situation de travail corporel peuvent créer des éléments de communication et d'expérience entre eux à partir d'un espace commun qui n'est pas celui de la parole, mais celui de la proprioception. Les réponses données autour des sensations liées au poids dans les exercices de décomposition des jambes et des bras indiquent ainsi qu'un espace commun d'expérience s'est établi autour de l'association membre relâché-sensation de légèreté. Cet espace commun pourrait être lui-même l'objet d'une expérimentation : une ambiance générale est-elle dégagée et ressentie durant ces exercices? Nous pensons ici à la notion de mind, " d'état d'esprit » développée avec le $\mathrm{BMC}^{\circledR}$ autour de chaque tissu du corps, et qu'il est proposé de percevoir par le toucher et par le mouvement (BAINBRIDGE-COHEN, 1993).

Ce commun fait référence à ce dont parle Marcelo de Andrade Pereira (2018), à partir d'Agamben et de Todorov : l'espace de relation privilégié, non pour déterminer de manière rigide les liens entre l'individuel et ce qui est commun, mais pour nous donner « [...] les moyens de penser la relation de l'individuel avec ce qui est commun, l'inscription du différent dans le même, de l'asymétrique en lien au symétrique » (PEREIRA, 2018, p.39) $)^{18}$.

teur,

En ce sens, l'expérience collective concerne ici un commun qui, selon l'au-

[...] se constitue comme ce lieu à partir duquel toutes les appartenances possibles deviennent susceptibles d'être fondées ; cela ne veut pas dire, néanmoins, que le commun puisse être réduit à une structure de liaison ou même fusionnelle (AGAMBEN, 2013). Le commun se trouve, pour Agamben, dans un registre différent de celui évoqué par le social, qui se caractérise, selon cette compréhension conceptuelle, comme un espace de symétrie pleine, un espace collectif (PEREIRA, 2018, p. 38) ${ }^{19}$.

Différent, ainsi, de l'acception symétrique donnée d'habitude à l'espace collectif, le travail présenté ici ne prend pas en considération les relations sociales, mais l'expérience du corps comme fondement du collectif, et ainsi d'un commun, tel que Pereira le décrit et selon lequel sétablit un « [...] autre plan de relations où l'Autre peut intervenir de fait » (PEREIRA, 2018, p. 38) ${ }^{20}$.

En outre, il ne s'agit pas de penser cette expérience somatique comme une base pour déterminer ce qui appartient à l'espace proprioceptif ou ce qui constitue l'expérience collective. Bien mieux, il s'agit d'imaginer d'autres possibilités pour le corps dans le système scolaire, circonscrit à différentes dynamiques transitoires. Des dynamiques permettant d'approfondir ce que nous considérons comme connaissance et inclure dans cette compréhension les possibilités d'un corps qui se réintègre au mouvement d'autrui, au « [...] déplacement permettant, enfin, de récupérer la signification de quelque chose qui n'appartient qu'à ce qui ne se laisse pas appartenir, qui appartient à la non appartenance - laquelle ne constitue pas un genre en soi, mais un moment où l'ordinaire se donne à voir, s'expose »(PEREIRA, 2018, p. 41) ${ }^{21}$. 
Comment l'expérience individuelle rejoint l'expérience collective: une étude en milieu scolaire à partir du travail de François Delsarte

Ainsi, cette recherche nous encourage à imaginer, dans le milieu scolaire, l'expérience proprioceptive comme une puissance de création et comme une puissance favorisant le lien collectif, le commun, où élèves, professeurs et professeures peuvent trouver d'autres modalités de production de connaissance. Selon les mots de Fernando Barcena, « il n'existe pas d'apprentissage, alors, qui ne suppose pas un devenir présent dans les choses. Nous faire présents à quelque chose implique de s'arrêter, de stopper, de réduire la vitesse et de s'approcher de cette chose " (BARCENA, 2018, p. 714)22. Ce serait, grosso modo, l'un des déploiements possibles du travail sur la proprioception, l'une des contributions possibles du travail de Delsarte - et, plus amplement, de l'Éducation Somatique - au système éducatif scolaire.

\section{Références}

ALEXANDER, Gerda. L'eutonie: un chemin de développement personnel par le corps (3e éd. rev. et augm.). Paris : Tchou, 1996.

BAINBRIDGE-COHEN, Bonnie. Sensing, feeling and action. The Experimental Anatomy of Body-Mind Centering. Berkeley, Calif.: North Atlantic Books, 1993.

BARBA, Eugenio. A Canoa de Papel. Tratado de Anthropologia Teatral. São Paulo: Editora Hucitec, 1994.

BARCENA, Fernando. Filosofía de la educación: un aprendizaje. Educucação \& Realidade, Porto Alegre, v. 38, n. 3, p. 703-730, Sept. 2013. Disponible sur : <http://www.scielo.br/scielo. php?script=sci_arttext\&pid=S2175-62362013000300002\&lng=pt\&nrm=iso>. Consulté le 06 mars 2018.

BATSON, Glenna. Somatic Studies and Dance. International Association for Dance Medicine and Science, [s.1.], 17 set. 2009. Disponible sur : <www.iadms.org/resource/resmgr/resource_papers/somatic_studies.pdf >. Consulté le 12 janvier 2018.

BOLSANELLO, Débora. Em Pleno Corpo: Educação Somática, movimento e saúde. Curitiba: Juruá, 2010.

BOLSANELLO, Débora. A Educação Somática e os conceitos de descondicionamento gestual, autenticidade somática e tecnologia interna. Motrivivência, Florianopolis, ano XXII, n. 36, p. 306-322, jun. 2011.

CAMPIGNION, P., \& DENYS-STRUYF, G. Respir-actions: programme d'exercices et d'étude des mécanismes de la respiration. Paris: Éditions Frison-Roche, 2012.

CARRINGTON, Walter. Pensando en Voz Alta: charlas sobre la enseñanza de la Técnica Alexander. Berkeley: Mornum Time Press, 2009.

CORRÊA GRAVINA, Heloisa. Eu tenho um Corpo, eu sou um Corpo: abordagens somáticas do movimento na graduação em dança. Revista Brasileira de Estudos da Presença, Porto Alegre, v. 5, n. 1, p. 233-258, jan./abr. 2015. Disponible sur : http://www.seer.ufrgs.br/index.php/ presenca/article/view/47496/32542. Consulté le 12 janvier 2018.

DELSARTE, François. Nota pessoal Beau. In: François Alexandre Nicolas Delsarte Papers, Mss. 1301, Louisiana and Lower Mississippi Valley Collections, LSU Libraries, Baton Rouge, La. Baton Rouge. Box 9-10, folder OS 36a, document 3. Consulté en mai 2006b.

DELSARTE, François. Nota pessoal. In: Fonds de M. Serge Bouts (aujourd'hui décédé), 24 rue Chazelles, 75017, Paris, Dossier « Larynx », document 3 Consulté en mai 2005.

DELSARTE, François. Nota pessoal. In: François Alexandre Nicolas Delsarte Papers, Mss. 1301, Louisiana and Lower Mississippi Valley Collections, LSU Libraries, Baton Rouge, La. Baton Rouge. Box 1, folder 36a/items 9-14, document 3. Consulté en mai 2006a. 
DELSARTE, François. Nota pessoal. In: François Alexandre Nicolas Delsarte Papers, Mss. 1301, Louisiana and Lower Mississippi Valley Collections, LSU Libraries, Baton Rouge, La. Baton Rouge. Box 1, folder 36a/items 9-14, document 9. Consulté en mai 2006c.

DELSARTE, François. Dictée de 1869 no 5 Un célèbre comédien a dit. In: François Alexandre Nicolas Delsarte Papers, Mss. 1301, Louisiana and Lower Mississippi Valley Collections, LSU Libraries, Baton Rouge, La. Baton Rouge. Box 3, folder 154, document 2. 1869.

FERNANDES, Ciane. Quando o Todo é mais que a Soma das Partes: somática como campo epistemológico contemporâneo. Revista Brasileira de Estudos da Presença, Porto Alegre, v. 5, n. 1, p. 9-38, jan./abr. 2015. Disponible sur : http://www.seer.ufrgs.br/index.php/presenca/ article/view/47585/32478. Consulté le 13 janvier 2018.

GAUTHIER, Benoit C. Édition critique de manuscrits de François Delsarte et examen de leur pertinence épistémologique dans la formation d'un corps scénique moderne. Université du Québec à Montréal, 2011.

GODARD, Hubert. Le souffle, le lien. Marsyas, no 32, décembre 1994, p. 2731.

GRIFFITH, Ralph Thomas Hotchkin. The Hymns of the Rigveda, Kotagiri (Nilgiri) : [s.n.], 1896. Disponible sur : <http://www.sanskritweb.net/rigveda/griffith.pdf〉. Consulté le 25 sept. 2014.

HANNA, Thomas. Bodies in Revolt: a primer in somatic thinking. Novato : Freeperson Press, 1985 [1970].

HANNA, Thomas. The Field of Somatics. Somatics: Magazine-Journal of the Bodily Arts and Sciences, v. I, no1, autumn 1976, p. 30-34 Disponible sur : https://somatics.org/library/ htl-fieldofsomatics. Consulté le 22 novembre 2014.

HANNA, Thomas. The Body of Life. New York : Alfred A. Knopf, 1979.

HANNA, Thomas. What is Somatics? Somatics: Magazine-Journal of the Bodily Arts and Sciences, vol. V, no 4, Spring-Summer 1986, p. 4-8 Disponible sur : http://somatics.org/library/ htl-wis1.html. Consulté le 24 novembre 2014.

HANNA, Thomas. What is Somatics? IV. Somatics: Magazine-Journal of the Bodily Arts and Sciences, vol. VI, no 3, Autumn/Winter 1987-88. Disponible sur : http://somatics.org/library/ htl-wis4.html. Consulté le 23 novembre 2014.

HANNA, Thomas. Clinical Somatic Education: A New Discipline in the Field of Health Care. Somatics: Magazine-Journal of the Bodily Arts and Sciences, vol. VIII, no 1, Autumn/Winter 1990-91 Disponible sur : http://www.somatics.com/hannart.htm. Consulté le 21 novembre 2014.

LEABHART, Thomas (dir.). Essays on François Delsarte, Mime Journal 2004/2005. Claremont, California : Pomona College Theatre Department, 2005.

LIMA CAETANO, Patricia de. Por uma Estética das Sensações: o corpo intenso dos Bartenieff Fundamentals e do Body-Mind Centering. Revista Brasileira de Estudos da Presença, Porto Alegre, v. 5, n. 1, p. 206-232, Jan./Apr. 2015. Disponible sur : http://www.seer.ufrgs.br/ index.php/presenca/article/view/47495/32540. Consulté le 12 janvier 2018.

MACKAYE, Steele. Notebook of Mackaye studying with Delsarte no 3/7. In: François Alexandre Nicolas Delsarte Papers, Mss. 1301, Louisiana and Lower Mississippi Valley Collections, LSU Libraries, Baton Rouge, La. Baton Rouge. Box 12b, folder 7, document 3. Acessado em: maio 2006.

ORTIZ, P., ROUQUET, O., CAMPIGNION, P., CORNUT, G. Respiration et mouvement un voyage dans les éléments mis en jeu pendant la respiration. Pantin, France: Recherche en Mouvement, 2012 (vidéo). 
Comment l'expérience individuelle rejoint l'expérience collective: une étude en milieu scolaire à partir du travail de François Delsarte

PEREIRA, Marcelo de Andrade. Pedagogia Crítico-Performativa: tensionamentos entre o próprio e o comum no espaço-tempo escolar. Cadernos CEDES, Campinas, v.37, n.101, p.29-44, Avril. 2017. Disponible sur : <http://www.scielo.br/scielo.php?script=sci_arttext\&pid=S0101-32622017000100029\&lng=en\&nrm=iso >. Consulté le 06 mars 2018.

PIZARRO, Diego, CUNHA, Carla Sabrina. Mitopoiesis. Dança, educação somática e biologia celular. Brasília: Editora IFB, 2017.

PORTE, Alain. Francois Delsarte. Une anthologie. Paris : IPMC, 1992.

RANDI, Elena. L'estetica di François Delsarte: la dimensione spettacolare. Université de Padoue, 1992.

ROLL, Jean-Pierre. Physiologie de la kinesthèse. La proprioception musculaire : sixième sens ou sens premier? Intellectica, no 36-37, 2003, p. 49-66.

ROSSETO COSTA, Priscila, STRAZZACAPPA, Márcia. A quem possa Interessar: a Educação Somática nas pesquisas acadêmicas. Revista Brasileira de Estudos da Presença, Porto Alegre, v. 5, n. 1, p. 39-53, jan./abr. 2015. Disponible sur : http://www.seer.ufrgs.br/index.php/ presenca/article/view/47152/32507. Consulté le 12 janvier 2018.

SHAWN, Ted. Every Little Movement. A Book about François Delsarte, the Man and his Philosophy, his Science and Applied Aesthetics, the Application of this Science to the Art of the Dance, the Influence of Delsarte on American Dance. Pittsfield, Mass.: Eagle Print. and Binding Co., 1954.

THOMAS d'AQUIN. Somme théologique. Paris : Éditions du Cerf, 1999.

WAILLE, Franck, DAMOUR, Christophe. François Delsarte, une recherche sans fin. Paris : L'Harmattan, 2015.

WAILLE, Franck. Corps, arts et spiritualité chez François Delsarte (1811-1871). Des interactions dynamiques. Lille : ANRT, 2011 [2009].

WAILLE, Franck. La méthode somatique expressive de François Delsarte. Histoire, esthétique, anthropologie : de la neurophysiologie à la métaphysique. Montpellier: L'Entretemps, 2016.

\section{Notes}

${ }^{1}$ Cet article est le résultat d'un stage postdoctoral financé par la CAPES (Capes Fondation, Brésil) et mené en 2016-2017 dans le cadre du programme d'études supérieures en éducation à l'Universidade Federal do Rio Grande do Sul (Porto Alegre, Brésil), au sein du Grupo de Estudos em Educação, Teatro e Performance (GETEPE), sous la supervision de Gilberto Icle.

${ }^{2}$ Dans la version en anglais : « Somatics is the field which studies the soma: namely, the body as perceived from within by a first-person perception » (HANNA, 1986, p. 4) (nous traduisons).

${ }^{3}$ " Léducation somatique est venue de démarches individuelles, principalement faites par des artistes, qui avaient pour objectif de réussir leur propre rééducation par le biais de pratiques corporelles se présentant comme des alternatives aux traitements médicaux conventionnels [...]. Citons le cas de Feldenkrais, [...] qui avait des problèmes aux genoux, de Mathias Alexander, [...] un acteur qui avait perdu sa voix, ou encore de Mabel Todd [...], qui souffrait de paralysie durant son adolescence, pour ne citer que quelles-uns » (ROSSETO COSTA, STRAZZACAPPA, 2015, p. 44). Dans la version en portugais : «A Educação Somática surgiu da ação individual, principalmente de artistas, com o objetivo de alcançar sua própria reabilitação por meio de práticas corporais que se apresentavam como alternativas aos métodos de tratamento médico convencionais [...]. Reportemo-nos ao caso de Feldenkrais [...], que teve problemas no joelho, ao de $\mathrm{Ma}-$ thias Alexander [...], um ator que perdeu a voz, ou mesmo Mabel Todd [...], que teve paralisia durante 
sua adolescência, para citar apenas alguns » (ROSSETO COSTA, STRAZZACAPPA, 2015, p. 44) (nous traduisons).

${ }^{4}$ Dans la version en portugais : «[...] o sentido de si [...]» (LIMA CAETANO, 2015, p. 217) (nous traduisons).

${ }^{5}$ Dans la version en portugais : « integração entre sensação-sentimento-ação (COHEN, 1993) » (FERNANDES, 2015, p. 15) (nous traduisons).

${ }^{6}$ Dans la version en portugais : « [...] a somática se baseia em um contexto de aprendizado receptivo e perceptivo, facilitando a conexão sensorial através da pausa dinâmica e do refinamento do esforço muscular integrados no todo da pessoa e do ambiente »(FERNANDES, 2015, p. 13) ) (nous traduisons).

${ }^{7}$ Dans la version en portugais : « [...] uma técnica somática pode ser ensinada de modo totalmente incoerente, isto é, não somático. Esse seria o caso, por exemplo, de ensinar os Bartenieff Fundamentals ${ }^{\mathrm{TM}}$ como uma série de exercícios a serem realizados numa sequência preestabelecida e aprendida por imitação. Portanto, a coerência entre método e conteúdo é outra característica da somática, confirmando seu caráter integrado » (FERNANDES, 2015, 2015, p. 17) (nous traduisons).

${ }^{8}$ Dans la version en portugais : «[...] mas é importante conectar-se com ritmos pessoais que, muitas vezes, são perdidos ou atropelados nas rotinas e obrigações do dia a dia. Nesse contexto de redescoberta de seus próprios ritmos [...]» (FERNANDES, 2015, p. 22) (nous traduisons).

${ }^{9}$ Nous faisons un lien entre les notions de qualités du mouvement développées par Delsarte (cf. Waille, 2011, p. 472-482) et l'approche du BMC ${ }^{\circledR}$ qui favorise la perception des qualités et des minds (états d'esprit, ambiances) associés à tel ou tel tissu ou élément fin (cellules par exemple) de l'organisme (cf. PIZARRO, CUNHA, 2017).

${ }^{10}$ Dans la version en anglais : «A soma, then, is a body perceived from within » (HANNA, 1990-91) (nous traduisons).

${ }^{11}$ Dans la version en portugais : « [...] a Educação Somática tende a suscitar um processo de compreensão expandido para o despertar dos sentidos, para além da visão e do aprendizado pela reprodução e pela repetição »(PIZARRO, CUNHA, 2017, p. 22) (nous traduisons).

${ }^{12}$ Dans la version en portugais : « [...] sair de seus hábitos perceptivos, motores e conceituais [...]» (DANIS BOIS cité in BOLSANELLO, 2010, p. 12) (nous traduisons).

${ }^{13}$ La position libre des genoux, par l'alignement des os des jambes qui peuvent assurer tout leur rôle dans la verticalité, elle est la plus facilitante pour prendre conscience du travail du poids en soi, dans la mesure où le poids transite vers le sol par le système squelettique, et que c'est par les os aussi que, dans la position verticale, peut le mieux être ressenti la contre-réaction (verticalisation) qui se produit en écho au trajet du poids vers le sol.

14 Dans la version en portugais : «[...] sentido de si [...]» (LIMA CAETANO, 2015, p. 217) (nous traduisons).

${ }^{15}$ Dans la version en portugais : «É definitivamente questão de tempo; questão de tempo dado por parte do professor, questão de tempo a que se permite o aluno »(CARRIGTON, 2009, p. 57) (nous traduisons).

${ }^{16}$ Dans la version en portugais : «A força motivadora para o intelecto e também a reserva de conhecimento científico de buscas que são fonte de capacidade como uma facilitadora de ações para a prosperidade da sociedade (Hindu Revolution, 2011, s.p.)»(FERNANDES, 2015, p. 14) (nous traduisons). 
${ }^{17}$ Dans la version en portugais : « Nessa tradição cultural milenar, soma é também uma divindade e uma bebida responsável pela imortalidade, além de uma substância constitutiva de todos os seres viventes » (FERNANDES, 2015, p. 14) (nous traduisons).

${ }^{18}$ Dans la version en portugais : « [...] subsídios para que possa ser pensada a relação do próprio com o comum, a inscrição do diferente no mesmo, do assimétrico em relação ao simétrico » (PEREIRA, 2018, p. 39) (nous traduisons).

${ }^{19}$ Dans la version en portugais : « [...] constitui se como esse lugar a partir do qual todos os possíveis pertencimentos fazem se passíveis de serem fundados; isso não quer dizer, contudo, que o comum possa ser reduzido a uma estrutura vinculativa ou mesmo fusional (AGAMBEN, 2013). O comum está, para Agamben, em um registro diferente daquele invocado pelo social, que se caracteriza, desde essa notação conceitual, como um espaço de plena simetria, coletivo »(PEREIRA, 2018, p. 38) (nous traduisons).

${ }^{20}$ Dans la version en portugais: “[...] outro plano relacional no qual o Outro pode de fato intervir" (PEREIRA, 2018, p. 38) (nous traduisons).

${ }^{21}$ Dans la version en portugais: “[...] transitar [que] permite, por fim, recobrar a significação de algo que não pertence senão ao que não se deixa pertencer, que pertence ao não pertencimento — o qual não constitui em si um gênero, mas, antes, um momento no qual o qualquer dá se a ver, expõe se” (PEREIRA, 2018, p. 41) (nous traduisons).

${ }^{22}$ Dans la version en espagnol: « No hay aprendizaje, entonces, que no suponga un hacerse presente en las cosas. Hacernos presente en algo implica pararse, detenerse, aminorar la marcha y aproximarse a algo » (BARCENA, 2018, p. 714) (nous traduisons).

* Professor da Académie de Lyon, Lyon, França. Bolsista PNPD-CAPES no Programa de Pós-graduação em Educação da Universidade Federal do Rio Grande do Sul, Brasil.

** Doutor em Educação pela Universidade Federal do Rio Grande do Sul, Porto Alegre, Brasil. Professor no Programa de Pós-graduação em Educação da Universidade Federal do Rio Grande do Sul e no Programa de Pós-graduação em Artes Cênicas da Universidade de Brasília. Bolsista de Produtividade em Pesquisa nível 1-D do CNPq. 
Franck Waille - Gilberto Icle

\section{Correspondência}

Franck Waille - Chez Yves Waille, 54 chemin de la Sorbière, Le Villarnet, 38710 Lavars, France.

E-mail: franck.cw@gmail.com - gilbertoicle@gmail.com

Recebido em 19 de março de 2018

Aprovado em 04 de junho de 2018

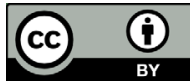

This work is licensed under a Creative Commons Attribution 4.0 International (CC BY 4.0). 